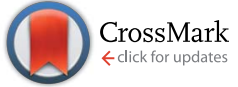

Cite this: RSC Adv., 2016, 6, 98344

\title{
Additive fabrication of nanostructures with focused soft X-rays $\dagger$
}

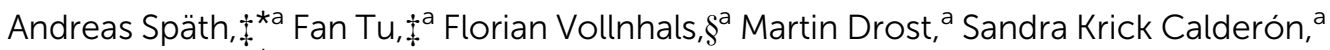 \\ Benjamin Watts, ${ }^{\text {b }}$ Rainer $\mathrm{H}$. Fink ${ }^{\text {ac }}$ and Hubertus Marbach ${ }^{\mathrm{a}}$
}

\begin{abstract}
We report on a novel technique for the fabrication of metallic nanostructures via soft X-ray irradiation of precursor molecules supplied from the gas phase. With this technique we were able to produce localized Co nanostructures with a growth rate and purity competitive with electron beam induced deposition. We demonstrate that our approach exhibits significant selectivity with respect to incident photon energy leading to enhanced growth for resonant absorption energy of the precursor molecule. Based on this finding we propose a unique new pathway of selective deposition from precursor mixtures. Furthermore, we investigated the growth rate with respect to precursor pressure and growth time and discuss the potential resolution limits of this new technique.
\end{abstract}

Received 17th July 2016

Accepted 10th October 2016

DOI: $10.1039 / c 6 r a 18214 c$

www.rsc.org/advances

precursor molecules supplied from the gas phase.$^{14-17}$ While the

\section{Introduction}

Progress in nanotechnology is driven by innovative processes for the controlled generation of functional nanostructures. The controlled deposition of metal nanostructures with well-defined purity and high aspect ratios plays an important role in the fabrication of nano-electronic and nano-magnetic devices, as well as novel optical elements for microscopy. ${ }^{\mathbf{1 - 6}}$ Lithographic techniques based on the irradiation of materials with photon or particle beams comprise a major class of methods for nanostructure fabrication. ${ }^{7-13}$ The most common forms of lithographic nanofabrication operate subtractively, radiochemically modifying the irradiated material so that it can be removed (or removes itself spontaneously), or so it resists removal relative to the unirradiated material, in further wet or dry-chemical processing and development steps. An alternative, additive form of lithographic nanofabrication involves using radiation to induce the local dissociation of precursor molecules and the subsequent deposition of one of the dissociation products. An example of such a technique is electron beam induced deposition (EBID), in which a focused electron beam is employed to induce the local dissociation of surface-adsorbed metal organic

\footnotetext{
aPhysikalische Chemie II and ICMM, Friedrich-Alexander-Universität Erlangen-Nürnberg (FAU), Egerlandstraße 3, 91058 Erlangen, Germany. E-mail: andreas.spaeth@fau.de

${ }^{b}$ Swiss Light Source (SLS), Paul Scherrer Institute, 5232 Villigen, Switzerland ${ }^{c}$ CENEM, Friedrich-Alexander-Universität Erlangen-Nürnberg (FAU), Egerlandstraße 3, 91058 Erlangen, Germany

$\dagger$ Electronic supplementary information (ESI) available. See DOI: $10.1039 /$ c6ra18214c

\$ These authors contributed equally to this work.

$\S$ Present address: Materials Research and Technology, Luxembourg Institute of Science and Technology (LIST), 41 rue du Brill, 4422 Belvaux, Luxembourg.
} non-volatile dissociation products remain as a deposit on the surface the volatile ones are pumped off the experimental chamber. ${ }^{14,18,19}$ The non-volatile portion usually contains the metal center of the precursor and forms a defined metalcontaining deposit that can be purified to almost clean metal nanostructures. ${ }^{20}$

Despite the various and promising applications of EBID, electron induced deposition processes with focused electron beams have an inherent drawback considering material selectivity. Since the primary energy of such a focused electron beam is usually well above $10 \mathrm{keV}$, all sorts of secondary and backscattered electrons are also generated. So even if the incident electron beam is monochromatic, the full energy range of electrons, from zero to the primary beam energy, is present in close proximity to the incident beam. Hence, one does not anticipate selectivity between different precursors even though the energy dependent cross section of electron induced precursor dissociation might vary between them. Therefore, precursors with comparable deposition rates are expected to generate mixed deposits when dosed simultaneously. In the present work this issue was addressed by replacing the incident electron beam by a focused beam of monochromatic X-rays.

Absorption of X-rays in the energy region of the $\mathrm{K}$ - and L-edges of the specimen leads to the formation of core holes and subsequent relaxation via fluorescence and Auger cascades. ${ }^{21,22}$ The radiochemical processes induced by X-ray beams - usually referred to as radiation damage - are mostly governed by interaction of the specimen with low energy secondary electrons produced by the Auger cascade. ${ }^{22}$ Therefore, the basic radiochemical processes are expected to be very similar for electron and X-ray irradiation, except that the latter requires a higher incident dose for a comparable quantitative 
effect. ${ }^{23}$ However, switching the primary irradiation from electrons to X-rays offers the possibility of resonant, and therefore chemically selective, excitation. For most materials, the X-ray absorption spectra tend to show distinctive discrete peaks in the near-edge energy region. This behaviour is often employed in near-edge X-ray absorption fine structure (NEXAFS) spectroscopy and resonant microscopic imaging and could be utilised to tailor the deposition rates by appropriate tuning of the incident photon energy. ${ }^{21,24}$ We therefore see the potential for sequential deposition of materials from various precursors without intermediate pumping and atmosphere exchange. This would be most useful for the fabrication of structures involving alternating multilayers, where a single precursor mixture could be used and the deposition material chosen by simply switching the incident photon energy.

The idea of focused X-ray beam induced deposition (FXBID) is based on the concept of X-ray lithography $y^{7,13,25,26}$ and has been successfully applied since the early 1990s for the generation of various metal and semiconductor thin films with moderate purity. ${ }^{27-30}$ The precursors investigated in these studies were basically the same metal organic complexes as commonly used in EBID. However, broad band synchrotron radiation was used due to the comparatively low brilliance of second generation synchrotrons. Therefore, the investigation of energy-selective deposition was not possible and it could not be excluded that the observed effects were mainly driven by the UV portion of the incident light. Furthermore, only large area thin films were deposited. X-ray stimulated electrochemical deposition of metal films from solution represents a similar approach, while also generating micro-patterned metal nanoparticle films. ${ }^{31-33}$ In the mid-1990s a photoninduced scanning Auger microscope was used to generate Pd and Mo deposits from standard precursors, but the resolution was still in the regime of several micrometers. ${ }^{34,35}$

The progress in instrumental development, and especially modern fabrication techniques for X-ray optics, ${ }^{4,36,37}$ have enabled the development of very efficient soft X-ray transmission spectro-microscopes (STXM) with $10 \mathrm{~nm}$ resolution. ${ }^{38}$ In STXM the incident X-ray beam is focused by a Fresnel zone plate and the specimen is raster-scanned through the focal spot with interferometric control while the transmitted photon energy is recorded. ${ }^{39}$ The PolLux-STXM ${ }^{40}$ at the Swiss Light Source enables also the implementation of a small gas flow around the sample position..$^{41}$ The respective gas cell is confined by two $50 \mathrm{~nm}$ thick $\mathrm{Si}_{3} \mathrm{~N}_{4}$-membranes that provide sufficient transmission over a broad photon energy range. Based on these unique opportunities, we present the novel generation of metal nanostructures via X-ray irradiation in an additive, direct write technique. A scheme of the modified STXM setup for FXBID studies and the interaction of the soft X-ray beam with the precursor molecules is depicted in Fig. 1. A major advantage of the use of a STXM setup for these investigations is the inherent possibility to characterize the deposits directly after generation by means of NEXAFS and high-resolution X-ray microscopy. ${ }^{42}$ Therefore, we are also able to present an analysis of deposition rates and chemical purity of the deposits fabricated with the novel FXBID technique.

\section{Experimental}

The precursor investigated during this proof-of-concept study was $\mathrm{Co}(\mathrm{CO})_{3} \mathrm{NO}$, which represents an intensely studied, standard precursor in EBID of Co-containing nanostructures. . $^{1,42,43}$ The residual pressure prior to precursor dosage was $\sim 4 \times 10^{-7}$ mbar. Note that within the current setup, the gas pressure is measured between the gas flux cell and the pumping system. Therefore, we assume slightly higher values within the gas flux cell than is recorded by the pressure gauge. All deposits presented within this work have been generated while scanning the PolLux piezo stages in constant velocity mode in order to achieve homogeneous deposition. The size of the deposits is $2 \times 1$ $\mu \mathrm{m}^{2}$, while a scan acceleration distance of $0.5 \mu \mathrm{m}$ was neglected on both sides of the fast scan direction in order to guarantee homogeneous irradiation of the specimen areas considered for quantitative analysis (effective size of deposit: $1 \times 1 \mu \mathrm{m}^{2}, 100 \times$ 100 acquisition points).

Considering the quantitative analysis of deposited mass versus incident photons, we characterized the efficiency of the detection setup (scintillator based photomultiplier tube) compared to a calibrated photodiode ( $c f$. ESI $\dagger$ ) and also corrected for the respective absorption of the $\mathrm{Si}_{3} \mathrm{~N}_{4}$-membranes to calculate the photon flux inside the gas cell. A detailed explanation of the thickness and material quantity evaluation can be found in ref. 42.

\section{Results and discussion}

Fig. 2a shows a STXM micrograph (optical density) of an array of FXBID deposits written with a precursor dosing pressure of 8.5 $\times 10^{-6}$ mbar. The irradiation time per pixel was varied from 20 to $80 \mathrm{~ms}$, while three different photon energies were investigated to analyze deposition rates well-below and above the absorption L-edge of Co (770.0 and $800.0 \mathrm{eV}$, respectively) as well as at the resonant excitation energy $(780.0 \mathrm{eV})$. Note that the maximum gas pressure of $\mathrm{Co}(\mathrm{CO})_{3} \mathrm{NO}$ was too low to record an absorption spectrum of the precursor in the gas phase. Therefore, the resonant energy was chosen close to the absorption maximum of the resulting Co deposits. We propose that the energy dependent effects discussed below could be further increased with an optimized value for the precursor absorption resonance. It is directly visible from the different brightness levels present in the micrograph and further illustrated by linear profiles across the deposits (Fig. 2b) that the optical density, which correlates with the effective thickness of Co within the deposits, is directly proportional to the irradiation time per pixel. Furthermore, we find a non-linear dependence of deposited Co quantity and incident photon energy. While the $800 \mathrm{eV}$ deposits are just slightly thicker than their pre-resonant analogues, resonant excitation at $780 \mathrm{eV}$ leads to an enhanced deposition rate. It should be further noted that the deposits are spatially well-defined and do not exhibit pronounced proximity effects as is often found in standard EBID experiments. ${ }^{44}$

A careful analysis of the energy dependent deposition rates requires calibration to the incident photon flux, detection efficiency ( $c f$. ESI $\dagger$ ) and absorption of the $\mathrm{Si}_{3} \mathrm{~N}_{4}$-membranes of the 


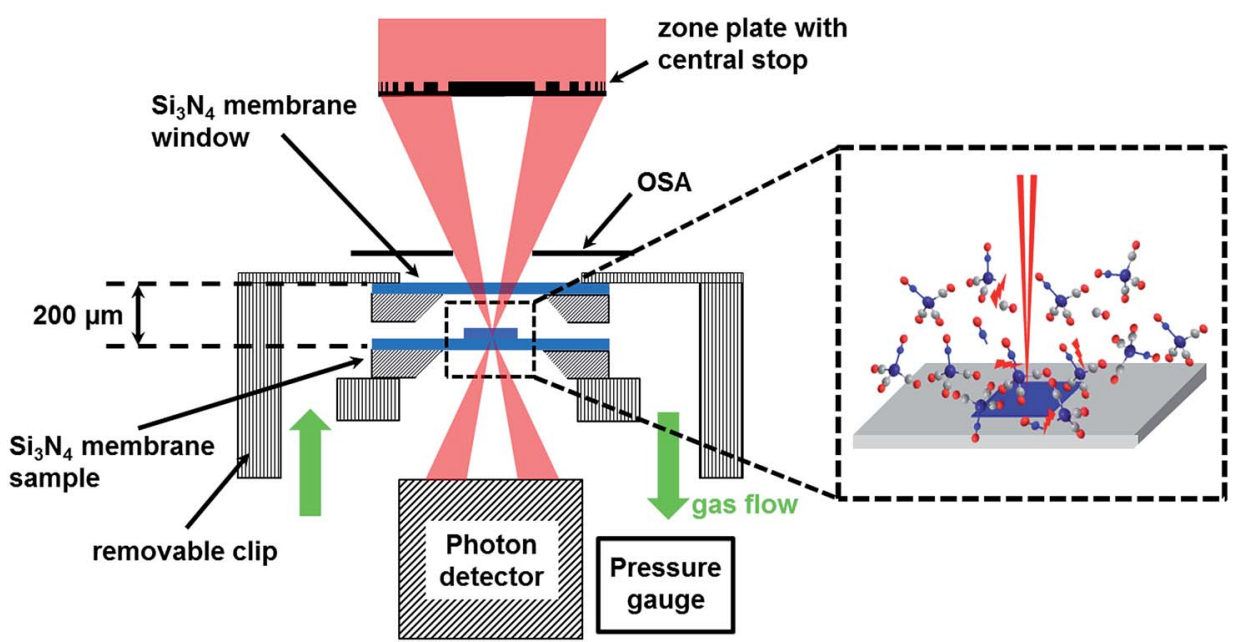

Fig. 1 Scheme of FXBID and the interaction of synchrotron $\mathrm{X}$-ray beam with $\mathrm{Co}(\mathrm{CO})_{3} \mathrm{NO}$ precursors on $\mathrm{Si}_{3} \mathrm{~N}_{4}$ membrane surface.

gas cell. Especially the consideration of incident photon flux is crucial for the determination of absolute growth rates, since it may vary strongly with the photon energy. With respect to these corrections, the amount of deposited Co can be compared to the number of incident photons (directly proportional to irradiation time). The result of this analysis is depicted in Fig. 3a. We find a clear linear dependence of effective Co thickness with illumination time per pixel and significant energy selectivity. The deposition rate at the resonant energy is $20 \%$ higher than for the pre-edge energy $(770 \mathrm{eV})$ and almost $40 \%$ higher compared to the post-edge energy $(800 \mathrm{eV})$. According to the standard models of X-ray induced radiochemistry we expect a correlation of radiation damage and the absorption cross section of the precursor molecule within this energy regime. ${ }^{45}$ Therefore, it is not surprising that the resonant energy exhibits higher deposition rates. However, the post-edge rate would be expected to be higher than the pre-edge rate. As discussed above, the exact absorption spectrum of the precursor molecule is not known and pre-edge absorption peaks might contribute to the observed behaviour. ${ }^{46}$ In addition, energy dependent radiation damage pathways cannot be fully excluded. ${ }^{47}$ The increased deposition rate at the resonant photon energy, however, provides access to a unique path of energy-selective deposition from precursors with different metal centres. As long as the general stability of a set of precursors is comparable, the deposition rate should be governed only by their characteristic X-ray absorption cross sections with discrete resonance energies. A certain precursor will not be significantly affected by the focused X-ray beam far below or above its absorption edge during the resonant deposition of a different metal.

Considering absolute numbers we find that about 2000 incident photons are required for the deposition of one Co atom. Although this appears to be a quite low efficiency, the deposition times of our FXBID approach ( $\sim 15 \mathrm{~min}$ for the $80 \mathrm{~ms}$ deposits) are competitive to EBID growth times of comparable structures. ${ }^{42}$ The seemingly low quantum yield for dissociation
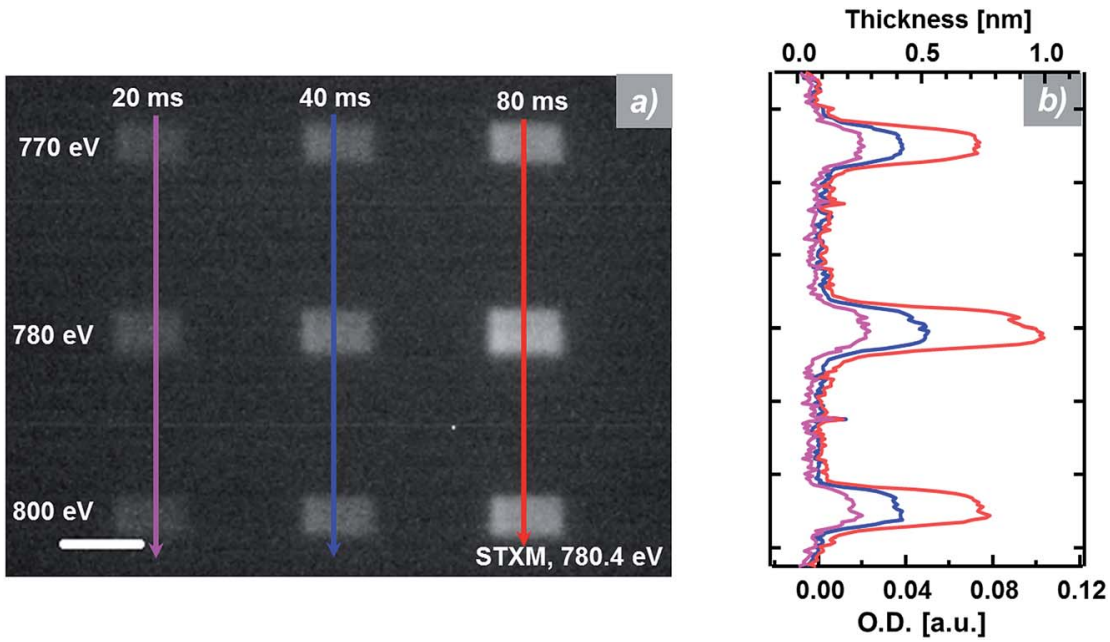

Fig. 2 (a) Resonant STXM micrograph of Co deposits from FXBID at varied irradiation time per pixel (columns) and photon energy (rows). (b) Profiles across the deposits illustrate the variations of the resulting optical density (proportional to quantity of deposited Co). 

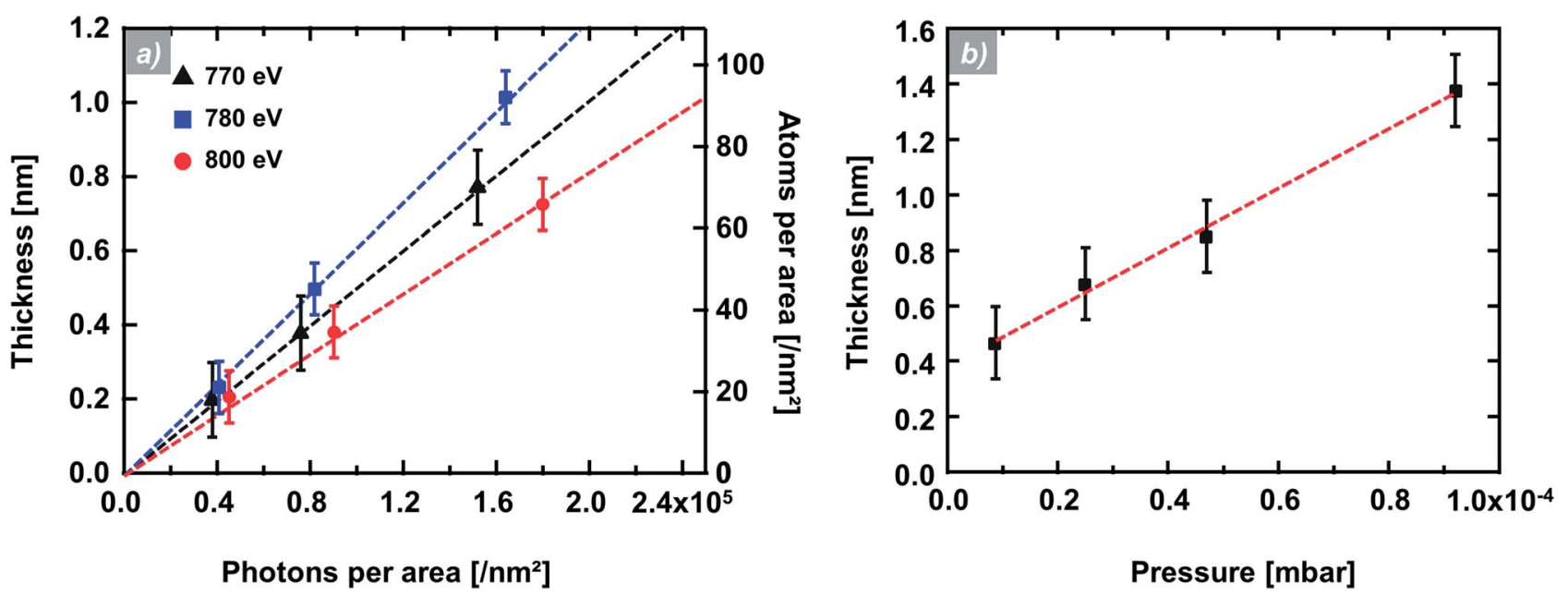

Fig. 3 (a) Quantitative analysis of the FXBID deposits depicted in Fig. 1. Effective Co thickness and correlated number of atoms per nm ${ }^{2}$ is compared to the calibrated amount of impinging photons per area during irradiation. (b) Dependence of effective Co thickness in FXBID deposits and precursor pressure. Deposition parameters: $40 \mathrm{~ms}$ irradiation time and $780.0 \mathrm{eV}$ incident photon energy.

can be comprehended by considering a low cross section for the process in combination with the relatively low gas pressure resulting a low molecular density on or close to the surface. Therefore, a large number of incident photons does not contribute to deposit formation even though the dissociation energies for the carbonyls in $\mathrm{Co}(\mathrm{CO})_{3} \mathrm{NO}$ are all below $10 \mathrm{eV} \cdot{ }^{48} \mathrm{In}$ perspective, more brilliant light sources with several magnitudes higher photon rates are already under development and should offer higher deposition rates. ${ }^{49,50}$

Additional insight into the deposition mechanism is gained by evaluating the growth rate with respect to the supply of fresh precursor (Fig. 3b). Therefore, we varied the precursor pressure within the gas cell over a range from $8.7 \times 10^{-6}$ to $9.2 \times 10^{-5}$ mbar. The latter value represents the highest achievable precursor pressure within our setup. We find again a linear dependence, suggesting that the deposition is not diffusion limited for the given experimental conditions. The linear fit of the growth rate does not tend to zero for very low precursor pressures. This finding might hint on a logarithmic increase of the growth rate below the $10^{-6}$ mbar regime.

An analysis of the purity of the FXBID deposits is presented by means of NEXAFS spectroscopy of an exemplary Co deposit in Fig. 4a. By fitting the FXBID deposit spectrum with two Gaussian peaks representing $\mathrm{Co}^{0}$ (extracted from the spectrum of a pure Co standard) and $\mathrm{Co}^{x+}$ (neglecting a detailed analysis of the various other oxidized states) we observe $\mathrm{Co}^{0}$ is the dominant oxidation state within these deposits. This finding is remarkable, considering that a comparable analysis of EBID deposits found mainly oxidized metal. ${ }^{42}$ However, in the present case we are able to perform spectroscopical investigations directly after deposition,
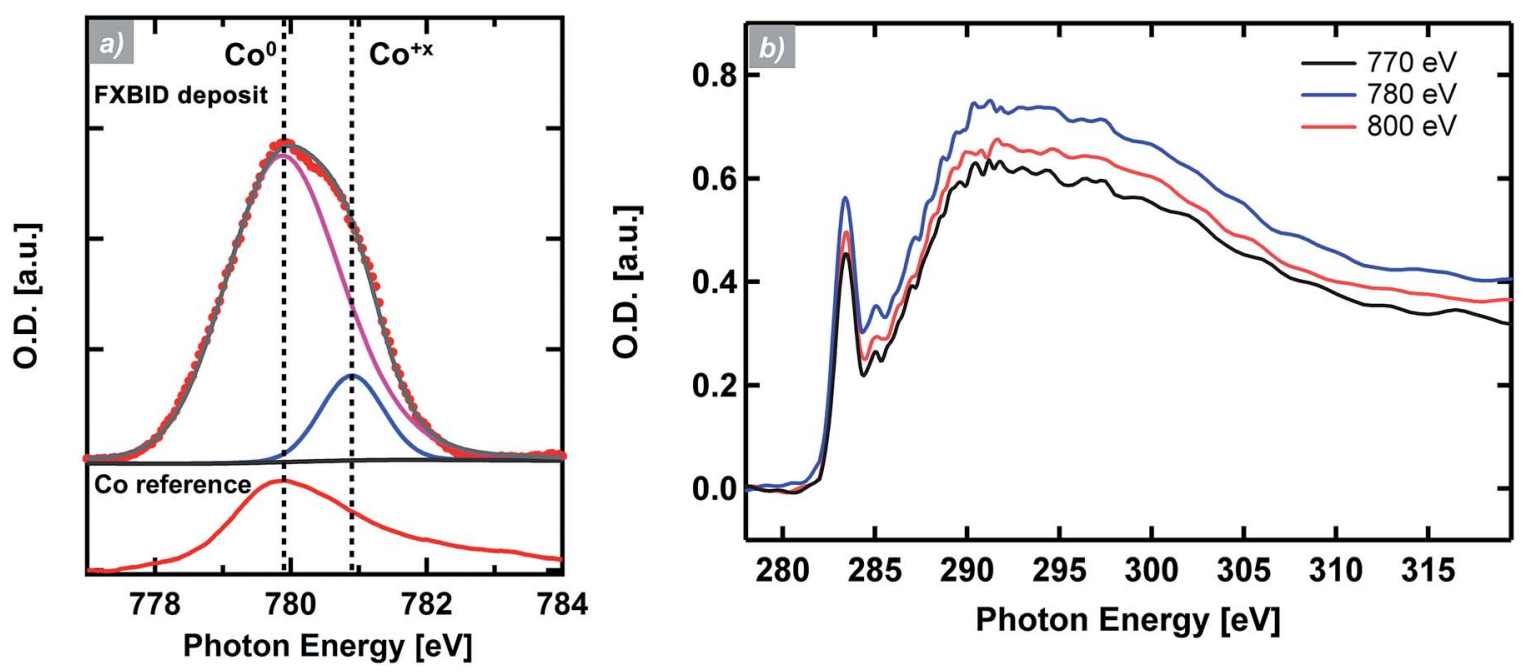

Fig. 4 (a) Co L-edge spectrum of FXBID deposit from $\mathrm{Co}(\mathrm{CO})_{3} \mathrm{NO}$ (photon energy: $780 \mathrm{eV}$; precursor pressure: $9.2 \times 10^{-5} \mathrm{mbar}$; illumination time per pixel: $40 \mathrm{~ms}$ ). Fitting with Gaussian peaks representing $\mathrm{CO}^{0}$ and $\mathrm{CO}^{x+}$ suggests only a minor contribution from oxidized states. (b) $\mathrm{C} \mathrm{K}$ edge spectra of FXBID deposits $\left(8.5 \times 10^{-5} \mathrm{mbar} ; 80 \mathrm{~ms}\right)$ at varied photon energy. 
while the respective EBID deposits could have been oxidized during transport to the synchrotron under ambient conditions. Fig. $4 \mathrm{~b}$ displays the $\mathrm{C}$ K-edge spectra of deposits generated under similar conditions. The spectral shape hints towards a crude mixture of many typical radiochemical end products containing a strong $\pi_{\mathrm{C}=\mathrm{C}}^{*}$-signal at about $285.0 \mathrm{eV}$ from unsaturated bonds. ${ }^{51}$ The amount of $\mathrm{C}$ correlates with the optical density at photon energies above the $\mathrm{C} \mathrm{K}$ edge jump (e.g. $320 \mathrm{eV}) .{ }^{24}$ Considering the three different photon energies, we find a similar quantitative trend compared to the amount of deposited Co. Resonant irradiation leads to an increased quantity of carbonaceous material within the deposits. Therefore, we can conclude that the carbon source within our experiment is mainly the precursor molecules and to a minor portion caused by residual gases. Although we find a significant amount of $\mathrm{C}$ within the deposits, the spectral analysis reveals a similar purity of FXBID deposits compared to EBID with higher amount of $\mathrm{Co}^{0}$ species. $^{42}$ In addition, the established purification techniques for EBID structures could be applied to our deposits, especially substrate heating, reactive gas mixtures and hydrogen radical treatment ${ }^{20}$ should be applicable with minor modifications of the present setup. Therefore, we conclude an overall competitive purity of FXBID deposits produced by the present technique. Additional studies will aim at the transition between autocatalytic and surface activated growth mechanism in FXBID, since these methods have been recently established in EBID for the production of comparably pure deposits with reduced proximity effects. ${ }^{42,52,53}$

Within these proof-of-concept studies, we did not include an investigation of FXBID spatial resolution limits so far. Since the maximum secondary electron energies that appear during soft FXBID are significantly lower than the high-energy backscattered electrons in EBID, ${ }^{\mathbf{1 4}}$ proximity effects should not play a major role in this technique. For the case of maskless, soft Xray lithographic patterning of thin polymer films, a spatial resolution slightly below $25 \mathrm{~nm}$ has previously been demonstrated. ${ }^{54}$ This value is close to the resolution limit of the zone plate employed during that study (outermost zone width: $\Delta r_{\mathrm{N}}=$ $15 \mathrm{~nm}$ ). Therefore it is expected that the resolution of soft X-ray direct write techniques is presently limited by the resolution of the applied focusing optics rather than methodological restrictions. Since resolution improvement of X-ray optics is of crucial interest to the X-ray microscopy community, it is currently being addressed by many promising approaches that are aiming for sub-10 $\mathrm{nm}$ resolution..$^{37,55,56}$

\section{Conclusions}

In conclusion, we have presented first results of a novel approach for additive nano-fabrication via focused soft X-rays and adsorbed molecular precursors. The growth rates and chemical purity of the fabricated deposits are competitive with the well-established EBID technique. The major advantage of the new technique is its energy selectivity of the molecular decomposition that is mainly driven by the X-ray absorption cross section at the transition metal L-edge of the metal-organic precursor molecule. We propose that precursors with different metal centers and comparable stability (as derived from growth rate in EBID) can be selectively addressed by
FXBID, resulting in the generation of structured bilayers, or even more complicated nanostructures, without intermediate pumping cycles. The resolution limit of FXBID has still to be evaluated in detail, but is expected to be limited by the resolution of the $\mathrm{X}$ ray optics of the STXM. Future studies will focus on a deeper mechanistic understanding of FXBID processes by precursor variation, substrate temperature variation and multi-sweep experiments addressing growth kinetics. Therefore, the present experimental flow cell has to be improved to allow the measurement of pressure and temperature directly within the gas cell. In addition, a method to optimize photon energy to maximum deposition rates has to be implemented. Ongoing experiments from our laboratories are evaluating the energy-selective deposition from precursor mixtures in detail.

\section{Acknowledgements}

We gratefully acknowledge experimental support by Dr Jörg Raabe and Blagoj Sarafimov from the PolLux beamline at the Swiss Light Source (SLS) and Markus Meyer (FAU). We thank Prof. Hans-Peter Steinrück (FAU) for support and fruitful discussions. Bernd Kress (FAU) gratefully contributed to a new precursor dosing system compatible to the PolLux gas flux cell. A. S., F. V. and S. K. C. acknowledge support by the Graduate School Molecular Science (GSMS). R. H. F. acknowledges support by the Deutsche Forschungsgemeinschaft (DFG) within the research training group "In situ microscopy" (GRK1896). H. M. acknowledges support through DFG grant MA 4246/1-2, cluster of excellence "Engineering of Advanced Materials" (EAM), COST action 1301 CELINA and MSCA-ITN 2016 ELENA. PolLux has been supported by the German Minister of Education and Research (BMBF, contract 05 K16 WED).

\section{Notes and references}

1 P. N. Bartlett, J. J. Baumberg, S. Coyle and M. E. Abdelsalam, Faraday Discuss., 2004, 125, 117.

2 K. W. Chou, A. Puzic, H. Stoll, G. Schütz, B. Van Waeyenberge, T. Tyliszczak, K. Rott, G. Reiss, H. Brückl and I. Neudecker, J. Appl. Phys., 2006, 99, 08 F305.

3 J. De Teresa and A. Fernández-Pacheco, Appl. Phys. A, 2014, 117, 1645.

4 I. Mohacsi, P. Karvinen, I. Vartiainen, V. A. Guzenko, A. Somogyi, C. M. Kewish, P. Mercere and C. David, J. Synchrotron Radiat., 2014, 21, 497.

5 O. V. Dobrovolskiy, M. Kompaniiets, R. Sachser, F. Porrati, C. Gspan, H. Plank and M. Huth, Beilstein J. Nanotechnol., 2015, 6, 1082.

6 P. Wohlhüter, M. T. Bryan, P. Warnicke, S. Gliga, S. E. Stevenson, G. Heldt, L. Saharan, A. K. Suszka, C. Moutafis, R. V. Chopdekar, J. Raabe, T. Thomson, G. Hrkac and L. J. Heyderman, Nat. Commun., 2015, 6, 7836.

7 M. Gentili, C. Giovannella and S. Selci, Nanolithography: A Borderland Between STM, EB, IB, and X-Ray Lithographies, Springer Science \& Business Media, Dordrecht, 1994.

8 T. Ito and S. Okazaki, Nature, 2000, 406, 1027.

9 A. A. Tseng, Small, 2005, 1(10), 924. 
10 I. Utke, S. Moshkhalev and P. Russell, Nanofabrication Using Focused Ion and Electron Beams, Oxford University Press, New York, 2012.

11 J. Fischer and M. Wegener, Laser Photonics Rev., 2013, 7(1), 22.

12 V. R. Manfrinato, L. Zhang, D. Su, H. Duan, R. G. Hobbs, E. A. Stach and K. K. Berggren, Nano Lett., 2013, 13(4), 1555.

13 J. R. Maldonado and M. Peckerar, Microelectron. Eng., 2016, 161, 87.

14 W. F. van Dorp and C. Hagen, J. Appl. Phys., 2008, 104, 081301.

15 I. Utke and A. Gölzhäuser, Angew. Chem., Int. Ed., 2010, 49, 9328.

16 C. Hagen, Appl. Phys. A, 2014, 117, 1599.

17 W. van Dorp, Appl. Phys. A, 2014, 117, 1615.

18 R. M. Thorman, R. Kumar, D. H. Fairbrother and O. Ingólfsson, Beilstein J. Nanotechnol., 2015, 6, 1904.

19 J. Postler, M. Renzler, A. Kaiser, S. E. Huber, M. Probst, P. Scheier and A. E. Ellis, J. Phys. Chem. C, 2015, 119, 20917.

20 A. Botman, J. Mulders and C. Hagen, Nanotechnology, 2009, 20, 372001.

21 J. Stöhr, NEXAFS Spectroscopy, Springer, Berlin, 1992.

22 J. Cazaux, J. Microsc., 1997, 188(2), 106.

23 A. P. Hitchcock, J. J. Dynes, G. Johansson, J. Wang and G. Botton, Micron, 2008, 39, 741.

24 H. Ade and A. P. Hitchcock, Polymer, 2008, 49, 643.

25 D. L. Spears and H. I. Smith, Electron. Lett., 1972, 8(4), 102.

26 R. Feder, E. Spiller and J. Topalian, Polym. Eng. Sci., 1977, 17(6), 385.

27 R. Zanoni, M. N. Piancastelli, J. McKinley and G. Margaritondo, Appl. Phys. Lett., 1989, 55, 1020.

28 D. C. Mancini, S. Varma, J. K. Simons, R. A. Rosenberg and P. A. Dowben, J. Vac. Sci. Technol., B: Microelectron. Process. Phenom., 1990, 8(6), 1804.

29 J. Takahashi, Y. Utsumi, H. Akazawa, I. Kawashima and T. Urisu, Appl. Phys. Lett., 1991, 58, 2776.

30 T. Ogata, S. I. Gheyas, H. Ogawa and M. Nishio, Thin Solid Films, 1995, 266, 168.

31 P. H. Borse, J. M. Yi, J. H. Je, S. D. Choi, Y. Hwu, P. Ruterana and G. Nouet, Nanotechnology, 2004, 15, S389.

32 P. C. Hsu, C. H. Wang, T. Y. Yang, Y. K. Hwu, C. S. Lin, C. H. Chen, L. W. Chang, S. K. Seol, J. H. Je and G. Margaritondo, J. Vac. Sci. Technol., A, 2007, 25(3), 615.

33 P. C. Hsu, Y. S. Chen, Y. K. Hwu, J. H. Je, G. Margaritondo and E. S. Tok, J. Synchrotron Radiat., 2015, 22, 1524.

34 P. Väterlein, M. Weiss, V. Wüstenhagen and E. Umbach, Appl. Surf. Sci., 1993, 70/71, 278.

35 P. Väterlein, V. Wüstenhagen and E. Umbach, Appl. Phys. Lett., 1995, 66, 2200.

36 J. Vila-Comamala, K. Jefimovs, J. Raabe, T. Pilvi, R. H. Fink, M. Senoner, A. Maaßdorf, M. Ritala and C. David, Ultramicroscopy, 2009, 109, 1360.
37 S. Werner, S. Rehbein, P. Guttmann and G. Schneider, Nano Res., 2014, 7(4), 528.

38 W. Chao, P. Fischer, T. Tyliszczak, S. Rekawa, E. Anderson and P. Naulleau, Opt. Express, 2012, 20(9), 9777.

39 A. L. D. Kilcoyne, T. Tyliszczak, W. F. Steele, S. Fakra, P. Hitchcock, K. Franck, E. Anderson, B. Harteneck, E. G. Rightor, G. E. Mitchell, A. P. Hitchcock, L. Yang, T. Warwick and H. Ade, J. Synchrotron Radiat., 2003, 10, 125.

40 J. Raabe, G. Tzvetkov, U. Flechsig, M. Böge, A. Jaggi, B. Sarafimov, M. G. C. Vernooij, T. Huthwelker, H. Ade, D. Kilcoyne, T. Tyliszczak, R. H. Fink and C. Quitmann, Rev. Sci. Instrum., 2008, 79, 113704.

41 T. Huthwelker, V. Zelenay, M. Birrer, A. Krepelova, J. Raabe, G. Tzvetkov, M. G. C. Vernooij and M. Ammann, Rev. Sci. Instrum., 2010, 81, 113706.

42 F. Vollnhals, M. Drost, F. Tu, E. Carrasco, A. Späth, R. H. Fink, H.-P. Steinrück and H. Marbach, Beilstein J. Nanotechnol., 2014, 5, 1175.

43 G. C. Gazzadi, H. Mulders, P. Trompenaars, A. Ghirri, M. Affronte, V. Grillo and S. Frabboni, J. Phys. Chem. C, 2011, 115, 19606.

44 W. F. van Dorp, S. Lazar, C. W. Hagen and P. Kruit, J. Vac. Sci. Technol., B: Microelectron. Nanometer Struct.-Process., Meas., Phenom., 2007, 25, 1603.

45 A. F. G. Leontowich, A. P. Hitchcock and R. F. Egerton, J. Electron Spectrosc. Relat. Phenom., 2016, 206, 58.

46 T. J. Regan, H. Ohldag, C. Stamm, F. Nolting, J. Lüning, J. Stöhr and R. L. White, Phys. Rev. B: Condens. Matter Mater. Phys., 2001, 64, 214422.

47 A. Späth, B. Watts, L. T. Wasserthal and R. H. Fink, J. Synchrotron Radiat., 2014, 21, 1153.

48 S. Engmann, M. Stano, P. Papp, M. J. Brunger, S. Matejčik and O. Ingólfsson, J. Chem. Phys., 2013, 138, 044305.

49 M. Eriksson, J. F. van der Veen and C. Quitmann, J. Synchrotron Radiat., 2014, 21, 837.

50 R. Hettel, J. Synchrotron Radiat., 2014, 21, 843.

51 A. F. G. Leontowich and A. P. Hitchcock, J. Vac. Sci. Technol., B: Nanotechnol. Microelectron.: Mater., Process., Meas., Phenom., 2012, 30(3), 030601.

52 H. Marbach, Appl. Phys. A, 2014, 117, 987.

53 F. Tu, M. Drost, F. Vollnhals, A. Späth, E. Carrasco, R. H. Fink and H. Marbach, Nanotechnology, 2016, 27, 355302.

54 A. F. G. Leontowich, A. P. Hitchcock, B. Watts and J. Raabe, Microelectron. Eng., 2013, 108, 5.

55 K. Keskinbora, C. Grévent, M. Bechtel, M. Weigand, E. Goering, A. Nadzeyka, L. Peto, S. Rehbein, G. Schneider, R. Follath, J. Vila-Comamala, H. Yan and G. Schütz, Opt. Express, 2013, 21(10), 11747.

56 I. Mohacsi, I. Vartiainen, M. Guizar-Sicairos, P. Karvinen, V. A. Guzenko, E. Müller, C. M. Kewish, A. Somogyi and C. David, Opt. Lett., 2016, 41(2), 281. 